Bull. Korean Math. Soc. 49 (2012), No. 4, pp. 885-898

http://dx.doi.org/10.4134/BKMS.2012.49.4.885

\title{
A NOTE ON SKEW DERIVATIONS IN PRIME RINGS
}

\author{
Vincenzo De FilipPis AND AJdA FošneR
}

\begin{abstract}
Let $m, n, r$ be nonzero fixed positive integers, $R$ a 2-torsion free prime ring, $Q$ its right Martindale quotient ring, and $L$ a non-central Lie ideal of $R$. Let $D: R \longrightarrow R$ be a skew derivation of $R$ and $E(x)=$ $D\left(x^{m+n+r}\right)-D\left(x^{m}\right) x^{n+r}-x^{m} D\left(x^{n}\right) x^{r}-x^{m+n} D\left(x^{r}\right)$. We prove that if $E(x)=0$ for all $x \in L$, then $D$ is a usual derivation of $R$ or $R$ satisfies $s_{4}\left(x_{1}, \ldots, x_{4}\right)$, the standard identity of degree 4 .
\end{abstract}

\section{Introduction}

Throughout, $R$ will represent an associative ring with a center $Z(R), Q$ its right Martindale quotient ring, and $C$ its extended centroid. Given an integer $n \geq 2$, a ring $R$ is said to be $n$-torsion free if for $x \in R, n x=0$ implies $x=0$. Recall that a ring $R$ is prime if for $a, b \in R, a R b=\{0\}$ implies that either $a=0$ or $b=0$, and is semiprime if $a R a=\{0\}$ implies $a=0$. As usual, the commutator $x y-y x$ will be denoted by $[x, y], x, y \in R$. An additive mapping $D: R \rightarrow R$ is called a derivation on $R$ if $D(x y)=D(x) y+x D(y)$ for all pairs $x, y \in R$. Let $a \in R$ be a fixed element. Then a map $D: R \rightarrow R$ defined by $D(x)=[a, x]=a x-x a, x \in R$, is a derivation on $R$. Such derivation is usually called an inner derivation defined by $a$.

Let $\alpha$ be an automorphism of a ring $R$. An additive mapping $D: R \rightarrow R$ is called an $\alpha$-derivation (or a skew derivation) on $R$ if $D(x y)=D(x) y+\alpha(x) D(y)$ for all pairs $x, y \in R$. In this case $\alpha$ is called an associated automorphism of $D$. Basic examples of $\alpha$-derivations are usual derivations and the map $\alpha-1$, where 1 denotes the identity map. Let $b \in Q$ be a fixed element. Then it is easy to see that a map $D: R \rightarrow R$ defined by $D(x)=b x-\alpha(x) b, x \in R$, is an $\alpha$-derivation called an inner $\alpha$-derivation (an inner skew derivation) defined by $b$. If a skew derivation $D$ is not inner, then it is outer.

An additive mapping $F: R \rightarrow R$ is called a generalized derivation on $R$ if there exists a derivation $D$ on $R$ such that $F(x y)=F(x) y+x D(y)$ for all pairs $x, y \in R$. Basic examples of generalized derivations are usual derivations on $R$, left $R$-module mappings from $R$ into itself, and so called generalized inner

Received April 28, 2011.

2010 Mathematics Subject Classification. 16W25, 16W20, 16N60.

Key words and phrases. skew derivation, automorphism, prime ring. 
derivations, i.e., maps of the form $x \mapsto a x+x b, x \in R$, where $a, b \in Q$ are fixed elements. Note also that generalized derivations and skew derivations are two natural generalizations of usual derivations.

We say that an automorphism $\alpha: R \rightarrow R$ is inner if there exists an invertible $q \in Q$ such that $\alpha(x)=q x q^{-1}$ for all $x \in R$. If an automorphism $\alpha \in \operatorname{Aut}(R)$ is not inner, then it is called outer.

Recently the following result was proved.

Theorem 1.1 ([6]). Let $m$ and $n$ be two fixed positive integers, $R$ a 2-torsion free prime ring, and $L$ a non-central Lie ideal of $R$. If

$$
F\left(x^{m+n+1}\right)=F(x) x^{m+n}+x^{m} D(x) x^{n}
$$

is an identity for $L$, where both $F$ and $D$ are generalized derivations of $R$, then either $D=0$ or $R$ satisfies the standard identity $s_{4}\left(x_{1}, \ldots, x_{4}\right)$ and $D$ is a usual derivation of $R$.

Let us point out that in [6, Theorem 1] the authors also considered the form of a generalized derivation $F$.

This result motivated us to investigate similar identity involving a skew derivation of a prime ring. More precisely, our aim is to prove the following theorem.

Theorem 1.2. Let $m, n, r$ be nonzero fixed positive integers, $R$ a 2-torsion free prime ring, $L$ a non-central Lie ideal of $R, D: R \longrightarrow R$ a skew derivations of $R$, and

$$
E(x)=D\left(x^{m+n+r}\right)-D\left(x^{m}\right) x^{n+r}-x^{m} D\left(x^{n}\right) x^{r}-x^{m+n} D\left(x^{r}\right), x \in R .
$$

If $E(x)=0$ for all $x \in L$, then $D$ is a usual derivation of $R$ or $R$ satisfies $s_{4}\left(x_{1}, \ldots, x_{4}\right)$, the standard identity of degree 4 .

\section{Preliminaries}

In this section we will write down some known results which we will need in the following.

Let $R$ be a prime ring and $I$ a two-sided ideal of $R$. Then $I, R$, and $Q$ satisfy the same generalized polynomial identities with coefficients in $Q$ (see [2]). Furthermore, $I, R$, and $Q$ satisfy the same generalized polynomial identities with automorphisms (Theorem 1 in [4]). Recall that in case $\operatorname{char}(R)=0$ an automorphism $\alpha$ of $Q$ is called Frobenius if $\alpha(x)=x$ for all $x \in C$. Moreover, in case $\operatorname{char}(R)=p \geq 2$ an automorphism $\alpha$ is Frobenius if there exists a fixed integer $t$ such that $\alpha(x)=x^{p^{t}}$ for all $x \in C$. In [4, Theorem 2] Chuang proved that if $\Phi\left(x_{i}, \alpha\left(x_{i}\right)\right)$ is a generalized polynomial identity for $R$, where $R$ is a prime ring and $\alpha \in \operatorname{Aut}(R)$ an automorphism of $R$ which is not Frobenius, then $R$ also satisfies the non-trivial generalized polynomial identity $\Phi\left(x_{i}, y_{i}\right)$, where $x_{i}$ and $y_{i}$ are distinct indeterminates. 
Now, let $R$ be a domain and $\alpha \in \operatorname{Aut}(R)$ an automorphism of $R$ which is outer. In [8] Kharchenko proved that if $\Phi\left(x_{i}, \alpha\left(x_{i}\right)\right)$ is a generalized polynomial identity for $R$, then $R$ also satisfies the non-trivial generalized polynomial identity $\Phi\left(x_{i}, y_{i}\right)$, where $x_{i}$ and $y_{i}$ are distinct indeterminates.

In [5] Chuang and Lee investigated polynomial identities with skew derivations. They proved that if $\Phi\left(x_{i}, D\left(x_{i}\right)\right)$ is a generalized polynomial identity for $R$, where $R$ is a prime ring and $D$ an outer skew derivation of $R$, then $R$ also satisfies the generalized polynomial identity $\Phi\left(x_{i}, y_{i}\right)$, where $x_{i}$ and $y_{i}$ are distinct indeterminates. Furthermore, they also proved [5, Theorem 1] that in the case $\Phi\left(x_{i}, D\left(x_{i}\right), \alpha\left(x_{i}\right)\right)$ is a generalized polynomial identity for $R$, where $R$ is a prime ring, $D$ an outer skew derivation of $R$, and $\alpha$ an outer automorphism of $R$, then $R$ also satisfies the generalized polynomial identity $\Phi\left(x_{i}, y_{i}, z_{i}\right)$, where $x_{i}, y_{i}$, and $z_{i}$ are distinct indeterminates.

Let us also mention that if $R$ is a prime ring satisfying a non-trivial generalized polynomial identity and $\alpha$ an automorphism of $R$ such that $\alpha(x)=x$ for all $x \in C$, then $\alpha$ is an inner automorphism of $R$ [1, Theorem 4.7.4].

For proving our main theorem we will also need the following lemma.

Lemma 2.1. Let $R$ be a prime ring of characteristic different from $2, m, n, r$ positive integers, and $0 \neq b \in R$ such that

$$
\left[r_{1}, r_{2}\right]^{m}\left(b\left[r_{1}, r_{2}\right]^{n}+\left[r_{1}, r_{2}\right]^{n} b\right)\left[r_{1}, r_{2}\right]^{r}=0
$$

for all $r_{1}, r_{2} \in R$. Then $R$ is commutative.

Proof. Firstly, assume that $b \in Z(R)$. In this case $R$ satisfies the generalized identity $2 b\left[x_{1}, x_{2}\right]^{m+n+r}=0$. Moreover, since $0 \neq b \in Z(R), R$ satisfies the polynomial identity $\left[x_{1}, x_{2}\right]^{m+n+r}=0$. By the result in [7] (for a bounded index on nilpotency), we conclude that $R$ must be commutative.

Now suppose that $b \notin Z(R)$. Then

$$
\left[x_{1}, x_{2}\right]^{m}\left(b\left[x_{1}, x_{2}\right]^{n}+\left[x_{1}, x_{2}\right]^{n} b\right)\left[x_{1}, x_{2}\right]^{r}=0
$$

is a non-trivial generalized polynomial identity for $R$. By Martindale's theorem [12], $R$ is a primitive ring having a nonzero socle with $C$ as the associated division ring. In light of Jacobson's theorem [9, p. 75], $R$ is isomorphic to a dense ring of linear transformations on some vector space $V$ over $C$. Let $\operatorname{dim}_{C} V \geq 3$. Since $b \notin C$, there exists $v \in V$ such that $\{v, b v\}$ are linearly $C$-independent. Moreover, because of the dimension of $V$ over $C$, there exists $w \in V$ such that $\{v, b v, w\}$ are linearly $C$-independent. By the density of $R$, there exist $r_{1}, r_{2} \in R$ such that

$$
r_{1} v=0, r_{2} v=w, r_{1} w=v, r_{1} b v=0, r_{2} b v=w .
$$

By calculation we obtain the contradiction

$$
0=\left[r_{1}, r_{2}\right]^{m}\left(b\left[r_{1}, r_{2}\right]^{n}+\left[r_{1}, r_{2}\right]^{n} b\right)\left[r_{1}, r_{2}\right]^{r} v=2 v \neq 0 .
$$


Hence, we may assume that $\operatorname{dim}_{C} V \leq 2$. So, either $R$ is commutative, or $R \cong M_{2}(C)$, i.e., the $2 \times 2$ matrix ring over $C$. We have to prove that if $R \cong M_{2}(C)$, then a contradiction follows.

Denote by $e_{i j}$ the usual unit matrix with 1 in the $(i, j)$-entry and zero elsewhere. Let $b=\sum_{1 \leq i, j \leq 2} b_{i j} e_{i j}$, where $b_{i j} \in C$. Recall that in case $\left[r_{1}, r_{2}\right] \neq 0$ for some $r_{1}, r_{2} \in M_{2}(C)$, then $\left[r_{1}, r_{2}\right]^{2} \in Z(R)$. More precisely, if $\left[r_{1}, r_{2}\right]$ is an invertible matrix, we have

$$
\left[r_{1}, r_{2}\right] b+b\left[r_{1}, r_{2}\right]=0 .
$$

Now consider $\left[r_{1}, r_{2}\right]=e_{11}-e_{22}$ in (1). By calculation we get $b_{11}=b_{22}=0$. Analogously, for $\left[r_{1}, r_{2}\right]=e_{12}+e_{21}$ in (1) we obtain $b_{12}+b_{21}=0$. On the other hand, for $\left[r_{1}, r_{2}\right]=e_{21}-e_{12}$ in (1) we have $b_{12}-b_{21}=0$. It follows that $b_{12}=b_{21}=0$. Thus, $b=0$, a contradiction.

We will end this section with one basic remark.

Remark 2.2. Our main assumption in Theorem 1.2 is

$$
D\left(x^{m+n+r}\right)=D\left(x^{m}\right) x^{n+r}+x^{m} D\left(x^{n}\right) x^{r}+x^{m+n} D\left(x^{r}\right)
$$

for all $x \in L$. On the other hand, the skew-derivation rule says that

$$
D\left(x^{m+n+r}\right)=D\left(x^{m}\right) x^{n+r}+\alpha\left(x^{m}\right) D\left(x^{n}\right) x^{r}+\alpha\left(x^{m+n}\right) D\left(x^{r}\right)
$$

for all $x \in R$. Therefore, by comparing (2) and (3) we get

$$
\left(\alpha\left(x^{m}\right)-x^{m}\right) D\left(x^{n}\right) x^{r}+\left(\alpha\left(x^{m+n}\right)-x^{m+n}\right) D\left(x^{r}\right)=0
$$

for all $x \in L$.

\section{The case of inner skew derivations}

In this section we will consider the case when $D: R \rightarrow R$ is a nonzero inner skew derivation on a prime $\operatorname{ring} R$ induced by the element $b \in Q$ and an automorphism $\alpha \in \operatorname{Aut}(R)$, that is, $D(x)=b x-\alpha(x) b$ for all $x \in R$. In this sense, our aim will be to prove the following proposition.

Proposition 3.1. Let $R$ be a prime ring of characteristic different from $2, L$ a non-central Lie ideal of $R, m, n, r \geq 1$ fixed integers, $b$ a nonzero element of $Q$, and $\alpha \in \operatorname{Aut}(R)$ an automorphism of $R$. If

$$
\left(\alpha\left(u^{m}\right)-u^{m}\right)\left(b u^{n}-\alpha\left(u^{n}\right) b\right) u^{r}+\left(\alpha\left(u^{m+n}\right)-u^{m+n}\right)\left(b u^{r}-\alpha\left(u^{r}\right) b\right)=0
$$

for all $u \in L$, then one of the following holds:
(a) $\alpha=1$, the identity map on $R$;
(b) $b x-\alpha(x) b=0$ for all $x \in R$;
(c) $R$ satisfies $s_{4}\left(x_{1}, \ldots, x_{4}\right)$.

We begin with the following lemma. 
Lemma 3.2. Let $R$ be a prime ring of characteristic different from $2, I$ a two-sided ideal of $R, m, n, r \geq 1$ fixed integers, $b$ a nonzero element of $Q, q$ an invertible element of $Q$, and $\alpha(x)=q x q^{-1}$ for all $x \in R$. If

$$
\left(\alpha\left(u^{m}\right)-u^{m}\right)\left(b u^{n}-\alpha\left(u^{n}\right) b\right) u^{r}+\left(\alpha\left(u^{m+n}\right)-u^{m+n}\right)\left(b u^{r}-\alpha\left(u^{r}\right) b\right)=0
$$

for all $u \in[I, I]$, then one of the following holds:

(a) $q \in C$ and hence $\alpha=1$, the identity map on $R$;

(b) $q^{-1} b \in C$ and hence $b x-\alpha(x) b=0$ for all $x \in R$;

(c) $R$ satisfies $s_{4}\left(x_{1}, \ldots, x_{4}\right)$.

Proof. By our assumption, $I$ satisfies

$$
\begin{gathered}
\left(q\left[x_{1}, x_{2}\right]^{m} q^{-1}-\left[x_{1}, x_{2}\right]^{m}\right)\left(b\left[x_{1}, x_{2}\right]^{n}-q\left[x_{1}, x_{2}\right]^{n} q^{-1} b\right)\left[x_{1}, x_{2}\right]^{r} \\
+\left(q\left[x_{1}, x_{2}\right]^{m+n} q^{-1}-\left[x_{1}, x_{2}\right]^{m+n}\right)\left(b\left[x_{1}, x_{2}\right]^{r}-q\left[x_{1}, x_{2}\right]^{r} q^{-1} b\right)=0 .
\end{gathered}
$$

Since $I$ and $Q$ satisfy the same generalized polynomial identities with automorphisms $Q$ also satisfies (5). Note that if $\left\{q^{-1} b, 1\right\}$ are linearly $C$-dependent, then $q^{-1} b \in C$ and we are done. Hence, consider the case when $\left\{q^{-1} b, 1\right\}$ are linearly $C$-independent. Then (5) is a non-trivial generalized polynomial identity for $Q$. By Martindale's theorem [12], $Q$ is a primitive ring having a nonzero socle with $C$ as the associated division ring. In a light of Jacobson's theorem [9, p. 75], $Q$ is isomorphic to a dense ring of linear transformations on some vector space $V$ over $C$. Of course, we may assume that $\operatorname{dim}_{C} V \geq 2$.

First, suppose that the vector space $V$ is finite dimensional over $C$, i.e., $\operatorname{dim}_{C} V=k \geq 2$. Then $Q \cong M_{k}(C)$, the ring of $k \times k$ matrices over $C$. We will denote by $b=\sum_{1 \leq i, j \leq k} b_{i j} e_{i j}$ and by $c=q^{-1} b=\sum_{1 \leq i, j \leq k} c_{i j} e_{i j}$ for $b_{i j}, c_{i j} \in C$.

Let $i \neq j$ and choose $\left[x_{1}, x_{2}\right]=e_{i i}-e_{j j}$ in (5). For all $t \neq i, j$ we have $e_{t t}\left(q\left(e_{i i}-e_{j j}\right)^{m} q^{-1}-\left(e_{i i}-e_{j j}\right)^{m}\right)\left(b\left(e_{i i}-e_{j j}\right)^{n}-q\left(e_{i i}-e_{j j}\right)^{n} q^{-1} b\right)\left(e_{i i}-e_{j j}\right)^{r} e_{t t}$ $+e_{t t}\left(q\left(e_{i i}-e_{j j}\right)^{m+n} q^{-1}-\left(e_{i i}-e_{j j}\right)^{m+n}\right)\left(b\left(e_{i i}-e_{j j}\right)^{r}-q\left(e_{i i}-e_{j j}\right)^{r} q^{-1} b\right) e_{t t}=0$.

Then

$$
q_{t i} c_{i t}+\gamma q_{t j} c_{j t}=0
$$

for all $i \neq j, t \neq i, j$, and $\gamma=(-1)^{m+n+r}$. Recall that for any $\varphi \in \operatorname{Aut}(Q)$

$$
\begin{aligned}
& \left(\varphi(q)\left[x_{1}, x_{2}\right]^{m} \varphi(q)^{-1}-\left[x_{1}, x_{2}\right]^{m}\right)\left(\varphi(b)\left[x_{1}, x_{2}\right]^{n}-\varphi(q)\left[x_{1}, x_{2}\right]^{n} \varphi(q)^{-1} \varphi(b)\right)\left[x_{1}, x_{2}\right]^{r} \\
& +\left(\varphi(q)\left[x_{1}, x_{2}\right]^{m+n} \varphi(q)^{-1}-\left[x_{1}, x_{2}\right]^{m+n}\right)\left(\varphi(b)\left[x_{1}, x_{2}\right]^{r}-\varphi(q)\left[x_{1}, x_{2}\right]^{r} \varphi(q)^{-1} \varphi(b)\right)=0
\end{aligned}
$$

is also an identity for $Q$. Therefore, the matrices $\varphi(q)$ and $\varphi(c)$ must satisfy the condition (6). In order to finish our proof we will use this argument a number of times.

In particular, let

$$
\begin{aligned}
& \varphi_{0}(x)=\left(1+e_{t i}\right) x\left(1-e_{t i}\right)=x+e_{t i} x-x e_{t i}-e_{t i} x e_{t i}, \\
& \varphi_{1}(x)=\left(1-e_{t i}\right) x\left(1+e_{t i}\right)=x-e_{t i} x+x e_{t i}-e_{t i} x e_{t i},
\end{aligned}
$$


and apply $(6)$ to $\varphi_{0}(q)$ and $\varphi_{0}(c)$. Then we have

$$
\left(q_{i i}-q_{t t}-q_{i t}\right) c_{i t}+\gamma q_{i j} c_{j t}=0
$$

for all $i \neq j$ and $t \neq i, j$. Analogously, applying (6) to $\varphi_{1}(q)$ and $\varphi_{1}(c)$ we obtain

$$
\left(-q_{i i}+q_{t t}-q_{i t}\right) c_{i t}-\gamma q_{i j} c_{j t}=0
$$

for all $i \neq j$ and $t \neq i, j$. Hence, by (7) and (8), and since $\operatorname{char}(R) \neq 2$, we have

$$
q_{i t} c_{i t}=0
$$

for all $i \neq t$. In the next step we will show that either $q$ is a diagonal matrix or $c$ is a diagonal matrix. So, suppose that $q$ is not diagonal. Then there exist integers $i \neq t$ such that $q_{i t} \neq 0$. By (9) it follows that $c_{i t}=0$.

Now, let $j \neq i, t$ and

$$
\begin{aligned}
& \chi_{0}(x)=\left(1+e_{i j}\right) x\left(1-e_{i j}\right)=x+e_{i j} x-x e_{i j}-e_{i j} x e_{i j}, \\
& \chi_{1}(x)=\left(1-e_{i j}\right) x\left(1+e_{i j}\right)=x-e_{i j} x+x e_{i j}-e_{i j} x e_{i j} .
\end{aligned}
$$

Denote $\chi_{0}(q)=\sum \chi(q)_{h l}^{\prime} e_{h l}, \chi_{1}(q)=\sum \chi(q)_{h l}^{\prime \prime} e_{h l}, \chi_{0}(c)=\sum \chi(c)_{h l}^{\prime} e_{h l}$, and $\chi_{1}(c)=\sum \chi(c)_{h l}^{\prime \prime} e_{h l}$. Here, $\chi(q)_{h l}^{\prime}, \chi(q)_{h l}^{\prime \prime}, \chi(c)_{h l}^{\prime}, \chi(c)_{h l}^{\prime \prime} \in C$. If both $\chi(q)_{i t}^{\prime}=0$ and $\chi(q)_{i t}^{\prime \prime}=0$, then $q_{i t}+q_{j t}=0=q_{i t}-q_{j t}$, which implies $q_{i t}=0$, a contradiction. Thus, at least one of $\chi(q)_{i t}^{\prime}$ and $\chi(q)_{i t}^{\prime \prime}$ is not zero. By applying (9), we have that either $\chi(c)_{i t}^{\prime}=0$ or $\chi(c)_{i t}^{\prime \prime}=0$. So, $0=c_{i t} \pm c_{j t}=c_{j t}$ and hence,

$$
q_{i t} \neq 0 \quad \Longrightarrow \quad c_{r t}=0
$$

for all $r \neq t$.

Consider $m \neq i, t$ and

$$
\begin{aligned}
& \mu_{0}(x)=\left(1+e_{t m}\right) x\left(1-e_{t m}\right)=x+e_{t m} x-x e_{t m}-e_{t m} x e_{t m}, \\
& \mu_{1}(x)=\left(1-e_{t m}\right) x\left(1+e_{t m}\right)=x-e_{t m} x+x e_{t m}-e_{t m} x e_{t m} .
\end{aligned}
$$

Denote $\mu_{0}(q)=\sum \mu(q)_{h l}^{\prime} e_{h l}, \mu_{1}(q)=\sum \mu(q)_{h l}^{\prime \prime} e_{h l}, \mu_{0}(c)=\sum \mu(c)_{h l}^{\prime} e_{h l}$, and $\mu_{1}(c)=\sum \mu(c)_{h l}^{\prime \prime} e_{h l}$ with $\mu(q)_{h l}^{\prime}, \mu(q)_{h l}^{\prime \prime}, \mu(c)_{h l}^{\prime}, \mu(c)_{h l}^{\prime \prime} \in C$. Then we can observe the following by $(10)$.

- If $0=\mu(q)_{i m}^{\prime}=q_{i m}-q_{i t}$, then $q_{i m} \neq 0$ and, by (10), $c_{r m}=0$ for all $r \neq m$.

- If $0=\mu(q)_{i m}^{\prime \prime}=q_{i m}+q_{i t}$, then $q_{i m} \neq 0$ and, by (10), $c_{r m}=0$ for all $r \neq m$.

- If both $\mu(q)_{i m}^{\prime} \neq 0$ and $\mu(q)_{i m}^{\prime \prime} \neq 0$, then by $(10)$, both $\mu(c)_{r m}^{\prime}=0$ and $\mu(c)_{r m}^{\prime \prime}=0$ for all $r \neq m$. In particular, for $r \neq t$ this means that $0=c_{r m}-c_{r t}$, and since $c_{r t}=0$ from (10) we have $c_{r m}=0$. On the other hand, for $r=t$ we have both $c_{t m}-c_{m m}+c_{t t}-c_{m t}=0$ and $c_{t m}+c_{m m}-c_{t t}-c_{m t}=0$. Since $c_{m t}=0$, by $(10)$ and $\operatorname{char}(R) \neq 2$, it follows that $c_{t m}=0$. 
Therefore, the previous step says that

$$
q_{i t} \neq 0 \quad \Longrightarrow \quad c_{r m}=0
$$

for all $r \neq m$ and $m \neq i$. In other words, if $q_{i t} \neq 0$, then the nonzero entries of the matrix $c$ are just on the $i$-th column and on the main diagonal.

Finally, let $j \neq i, t$ and

$$
\begin{aligned}
& \eta_{0}(x)=\left(1+e_{j i}\right) x\left(1-e_{j i}\right)=x+e_{j i} x-x e_{j i}-e_{j i} x e_{j i}, \\
& \eta_{1}(x)=\left(1-e_{j i}\right) x\left(1+e_{j i}\right)=x-e_{j i} x+x e_{j i}-e_{j i} x e_{j i} .
\end{aligned}
$$

Denote $\eta_{0}(q)=\sum \eta(q)_{h l}^{\prime} e_{h l}, \eta_{1}(q)=\sum \eta(q)_{h l}^{\prime \prime} e_{h l}, \eta_{0}(c)=\sum \eta(c)_{h l}^{\prime} e_{h l}$, and $\eta_{1}(c)=\sum \eta(c)_{h l}^{\prime \prime} e_{h l}$ with $\eta(q)_{h l}^{\prime}, \eta(q)_{h l}^{\prime \prime}, \eta(c)_{h l}^{\prime}, \eta(c)_{h l}^{\prime \prime} \in C$. Also we can observe the following by $(11)$.

- If $0=\eta(q)_{j t}^{\prime}=q_{j t}+q_{i t}$, then $q_{j t} \neq 0$ and, by (11), $c_{r i}=0$ for all $r \neq i$.

- If $0=\eta(q)_{j t}^{\prime \prime}=q_{j t}-q_{i t}$, then $q_{j t} \neq 0$ and, by (11), $c_{r i}=0$ for all $r \neq i$.

- If both $\eta(q)_{j t}^{\prime} \neq 0$ and $\eta(q)_{j t}^{\prime \prime} \neq 0$, then by (11) all the entries in the $i$-th column of $\eta_{0}(c)$ are zero. The same is true for the $i$-th column of $\eta_{1}(c)$. In particular, for $m \neq j$ this means that $0=\eta(c)_{m i}^{\prime}=c_{m i}-c_{m j}$, and since $c_{m j}=0$ from (11) we have $c_{m i}=0$. On the other hand, for $m=j$ we have both $0=\eta(c)_{j i}^{\prime}=c_{j i}+c_{i i}-c_{j j}-c_{i j}$ and $0=\eta(c)_{j i}^{\prime \prime}=$ $c_{j i}-c_{i i}+c_{j j}-c_{i j}$. Since $c_{i j}=0$, by $(11)$ and $\operatorname{char}(R) \neq 2$, it follows that $c_{j i}=0$.

This yields that if $q_{i t} \neq 0$, then the nonzero entries of the matrix $c$ are just on the main diagonal. The previous argument says that either $q$ is a diagonal matrix or $c$ is a diagonal matrix.

In the next step we will prove that either $q$ is a central matrix or $c$ is a central matrix. To do this, we assume first that $q$ is not a diagonal matrix. So, suppose that $q_{j i} \neq 0$ for some $i \neq j$. As above, we introduce some suitable automorphisms of $M_{k}(C)$. More precisely, let $m \neq i, j$ and

$$
\begin{aligned}
& \lambda_{0}(x)=\left(1+e_{i m}\right) x\left(1-e_{i m}\right)=x+e_{i m} x-x e_{i m}-e_{i m} x e_{i m}, \\
& \lambda_{1}(x)=\left(1-e_{m j}\right) x\left(1+e_{m j}\right)=x-e_{m j} x+x e_{m j}-e_{m j} x e_{m j} .
\end{aligned}
$$

Denote $\lambda_{0}(q)=\sum \lambda(q)_{h l}^{\prime} e_{h l}, \lambda_{1}(q)=\sum \lambda(q)_{h l}^{\prime \prime} e_{h l}, \lambda_{0}(c)=\sum \lambda(c)_{h l}^{\prime} e_{h l}$, and $\lambda_{1}(c)=\sum \lambda(c)_{h l}^{\prime \prime} e_{h l}$ with $\lambda(q)_{h l}^{\prime}, \lambda(q)_{h l}^{\prime \prime}, \lambda(c)_{h l}^{\prime}, \lambda(c)_{h l}^{\prime \prime} \in C$. Note that both $\lambda(q)_{j i}^{\prime}=q_{j i} \neq 0$ and $\lambda(q)_{j i}=q_{j i} \neq 0$. Therefore, both $\lambda_{0}(c)$ and $\lambda_{1}(c)$ are diagonal matrices. In particular,

$$
\begin{aligned}
& 0=\lambda(c)_{i m}^{\prime}=c_{m m}-c_{i i}, \\
& 0=\lambda(c)_{m j}^{\prime \prime}=c_{j j}-c_{m m},
\end{aligned}
$$

and hence, $c_{i i}=c_{j j}=c_{m m}$ and $c$ is a central matrix in $M_{k}(C)$.

Thus, we assume that $q$ is a diagonal matrix. Moreover, if there exists an automorphism $\theta$ of $M_{k}(C)$ such that $\theta(q)$ is not diagonal, then, by the previous argument, we can prove that $\theta(c)$ is central as well as $c$. Therefore, we may 
assume that $\theta(q)$ is a diagonal matrix for all $\theta \in \operatorname{Aut}\left(M_{k}(C)\right)$. In particular, let $l \neq t$ and

$$
\theta(x)=\left(1+e_{l t}\right) x\left(1-e_{l t}\right)=x+e_{l t} x-x e_{l t}-e_{l t} x e_{l t} .
$$

Denote $\theta(q)=\sum \theta(q)_{i j}^{\prime} e_{h l}$ with $\theta(q)_{i j}^{\prime} \in C$. Since $\theta(q)$ is diagonal, we have $\theta(q)_{l t}^{\prime}=0$. Hence, $q_{t t}-q_{l l}=0$. In this case we conclude that $q$ is a central matrix and we are done.

At the end, suppose that $\operatorname{dim}_{C} V=\infty$ and assume that $q \notin C$ and $c \notin C$. Under this assumption there exist $r_{1}, r_{2} \in Q$ such that $q r_{1} \neq r_{1} q$ and $c r_{2} \neq r_{2} c$. By Litoff's Theorem (see, for example, [10, p. 280]) there exist $e^{2}=e \in Q$ and a positive integer $k=\operatorname{dim}_{C}(V e)$ such that

$$
q, c, q r_{1}, r_{1} q, c r_{2}, r_{2} c, b, r_{1}, r_{2} \in e Q e \cong M_{k}(C) .
$$

Moreover, $e Q e$ satisfies the identity

$$
\left((e q e)\left[x_{1}, x_{2}\right]^{m}\left(e q^{-1} e\right)-\left[x_{1}, x_{2}\right]^{m}\right)\left((e b e)\left[x_{1}, x_{2}\right]^{n}-(e q e)\left[x_{1}, x_{2}\right]^{n}\left(e q^{-1} b e\right)\right)\left[x_{1}, x_{2}\right]^{r}
$$$$
+\left((e q e)\left[x_{1}, x_{2}\right]^{m+n}\left(e q^{-1} e\right)-\left[x_{1}, x_{2}\right]^{m+n}\right)\left((e b e)\left[x_{1}, x_{2}\right]^{r}-(e q e)\left[x_{1}, x_{2}\right]^{r}\left(e q^{-1} b e\right)\right)=0 .
$$

By the arguments in the previous case we have that either eqe $\in Z(e Q e)$ or $e c e=e q^{-1} b e \in Z(e Q e)$. Hence, one of the following holds:

- $q r_{1}=e q r_{1}=e q e r_{1}=r_{1} e q e=r_{1} q e=r_{1} q$,

- $c r_{2}=e c r_{2}=e c e r_{2}=r_{2} e c e=r_{2} c e=r_{2} c$.

In both cases we have a contradiction. The proof of lemma is completed.

Proof of Proposition 3.1. Set $I=R[L, L] R$. Then $0 \neq[I, R] \subseteq L$. Therefore, by our hypothesis

$$
\left(\alpha\left(u^{m}\right)-u^{m}\right)\left(b u^{n}-\alpha\left(u^{n}\right) b\right) u^{r}+\left(\alpha\left(u^{m+n}\right)-u^{m+n}\right)\left(b u^{r}-\alpha\left(u^{r}\right) b\right)=0
$$

for all $u \in[I, R]$. Since $I, R$, and $Q$ satisfy the same generalized polynomial identities with automorphisms it follows that $Q$ satisfies

$$
\begin{aligned}
& \left(\alpha\left(\left[x_{1}, x_{2}\right]^{m}\right)-\left[x_{1}, x_{2}\right]^{m}\right)\left(b\left[x_{1}, x_{2}\right]^{n}-\alpha\left(\left[x_{1}, x_{2}\right]^{n}\right) b\right)\left[x_{1}, x_{2}\right]^{r} \\
& +\left(\alpha\left(\left[x_{1}, x_{2}\right]^{m+n}\right)-\left[x_{1}, x_{2}\right]^{m+n}\right)\left(b\left[x_{1}, x_{2}\right]^{r}-\alpha\left(\left[x_{1}, x_{2}\right]^{r}\right) b\right)=0 .
\end{aligned}
$$

In the case $\alpha$ is inner, then there exists an invertible element $q \in Q$ such that $\alpha(x)=q x q^{-1}$ for all $x \in R$. Hence, by Lemma 3.2 the result follows.

Next, suppose that $\alpha$ is outer. Since $b \neq 0$, by the main theorem in [3], $Q$ satisfies a non-trivial generalized polynomial identity ( $Q$ is a GPI-ring). Therefore, by [12, Theorem 3] $Q$ is a primitive ring and it is a dense subring of the ring of linear transformations of a vector space $V$ over a division $\operatorname{ring} D$. Moreover, $Q$ contains nonzero linear transformations of finite rank.

If $\alpha$ is not Frobenius, then by [4, Theorem 2] and (12) we have that $Q$ satisfies

$$
\begin{gathered}
\left(\left[y_{1}, y_{2}\right]^{m}-\left[x_{1}, x_{2}\right]^{m}\right)\left(b\left[x_{1}, x_{2}\right]^{n}-\left[y_{1}, y_{2}\right]^{n} b\right)\left[x_{1}, x_{2}\right]^{r} \\
+\left(\left[y_{1}, y_{2}\right]^{m+n}-\left[x_{1}, x_{2}\right]^{m+n}\right)\left(b\left[x_{1}, x_{2}\right]^{r}-\left[y_{1}, y_{2}\right]^{r} b\right)=0
\end{gathered}
$$


and, in particular, $Q$ satisfies

$$
\left[x_{1}, x_{2}\right]^{m}\left(b\left[x_{1}, x_{2}\right]^{n}-\left[x_{1}, x_{2}\right]^{n} b\right)\left[x_{1}, x_{2}\right]^{r}=0 .
$$

Thus, $Q$ must be commutative from Lemma 2.1. On the other hand, if $Q$ is a domain, $Q$ satisfies both (13) and (14), and, as above, we conclude that $Q$ is commutative. In the light of previous arguments we assume that $\alpha$ is Frobenius and $\operatorname{dim}_{D} V \geq 2$. Note that if $\operatorname{char}(R)=0$, we have $\alpha(x)=x$ for all $x \in R$ since $\alpha$ is Frobenious. By [1, Theorem 4.7.4] this implies that $\alpha$ is inner, a contradiction. Thus, we may assume that $\operatorname{char}(R)=p>2$ and $\alpha(\gamma)=\gamma^{p^{t}}$ for all $\gamma \in C$ and some nonzero fixed integer $t$. In particular, $\alpha\left(\left[\gamma x_{1}, x_{2}\right]\right)=\gamma^{p^{t}} \alpha\left(\left[x_{1}, x_{2}\right]\right)$. Hence, by replacing $\left[x_{1}, x_{2}\right]$ with $\left[\gamma x_{1}, x_{2}\right]$ in (12) we obtain that $Q$ satisfies

$$
\begin{gathered}
\gamma^{m+n+r}\left(\gamma^{m\left(p^{t}-1\right)} \alpha\left(\left[x_{1}, x_{2}\right]^{m}\right)-\left[x_{1}, x_{2}\right]^{m}\right)\left(b\left[x_{1}, x_{2}\right]^{n}-\gamma^{n\left(p^{t}-1\right)} \alpha\left(\left[x_{1}, x_{2}\right]^{n}\right) b\right)\left[x_{1}, x_{2}\right]^{r} \\
+\gamma^{m+n+r}\left(\gamma^{(m+n)\left(p^{t}-1\right)} \alpha\left(\left[x_{1}, x_{2}\right]^{m+n}\right)\right)\left(b\left[x_{1}, x_{2}\right]^{r}-\gamma^{r\left(p^{t}-1\right)} \alpha\left(\left[x_{1}, x_{2}\right]^{r}\right) b\right) \\
-\gamma^{m+n+r}\left[x_{1}, x_{2}\right]^{m+n}\left(b\left[x_{1}, x_{2}\right]^{r}-\gamma^{r\left(p^{t}-1\right)} \alpha\left(\left[x_{1}, x_{2}\right]^{r}\right) b\right)=0
\end{gathered}
$$

for all $0 \neq \gamma \in C$. Since $\gamma \neq 0, Q$ satisfies

$$
\begin{gathered}
\left(\gamma^{m\left(p^{t}-1\right)} \alpha\left(\left[x_{1}, x_{2}\right]^{m}\right)-\left[x_{1}, x_{2}\right]^{m}\right)\left(b\left[x_{1}, x_{2}\right]^{n}-\gamma^{n\left(p^{t}-1\right)} \alpha\left(\left[x_{1}, x_{2}\right]^{n}\right) b\right)\left[x_{1}, x_{2}\right]^{r} \\
+\left(\gamma^{(m+n)\left(p^{t}-1\right)} \alpha\left(\left[x_{1}, x_{2}\right]^{m+n}\right)\right)\left(b\left[x_{1}, x_{2}\right]^{r}-\gamma^{r\left(p^{t}-1\right)} \alpha\left(\left[x_{1}, x_{2}\right]^{r}\right) b\right) \\
\text { (15) } \quad-\left[x_{1}, x_{2}\right]^{m+n}\left(b\left[x_{1}, x_{2}\right]^{r}-\gamma^{r\left(p^{t}-1\right)} \alpha\left(\left[x_{1}, x_{2}\right]^{r}\right) b\right)=0 .
\end{gathered}
$$

Since $Q$ is a primitive ring with a nonzero socle, by $[9$, p. 79$]$ there exists a semi-linear automorphism $T \in \operatorname{End}(V)$ such that $\alpha(x)=T x T^{-1}$ for all $x \in R$. Hence, by (15), $Q$ satisfies

$$
\begin{gathered}
\left(\gamma^{m\left(p^{t}-1\right)} T\left[x_{1}, x_{2}\right]^{m} T^{-1}-\left[x_{1}, x_{2}\right]^{m}\right)\left(b\left[x_{1}, x_{2}\right]^{n}-\gamma^{n\left(p^{t}-1\right)} T\left[x_{1}, x_{2}\right]^{n} T^{-1} b\right)\left[x_{1}, x_{2}\right]^{r} \\
+\left(\gamma^{(m+n)\left(p^{t}-1\right)} T\left[x_{1}, x_{2}\right]^{m+n} T^{-1}\right)\left(b\left[x_{1}, x_{2}\right]^{r}-\gamma^{r\left(p^{t}-1\right)} T\left[x_{1}, x_{2}\right]^{r} T^{-1} b\right) \\
\text { (16) } \quad-\left[x_{1}, x_{2}\right]^{m+n}\left(b\left[x_{1}, x_{2}\right]^{r}-\gamma^{r\left(p^{t}-1\right)} T\left[x_{1}, x_{2}\right]^{r} T^{-1} b\right)=0 .
\end{gathered}
$$

Denote the identity (16) by $\Phi\left(x_{1}, x_{2}\right)$. Assume first that $v$ and $T^{-1} b v$ are $D$ dependent for all $v \in V$. More precisely, let $T^{-1} b v=\lambda v$ for $\lambda \in D$. In this case

$$
\begin{aligned}
\left(b x-T x T^{-1} b\right) v & =b x v-T x T^{-1} b v=b x v-T(x(\lambda v))=b x v-T(\lambda(x v)) \\
& =b x v-T\left(T^{-1} b\right)(x v)=b x v-b x v=0
\end{aligned}
$$

for all $x \in R$. This yields that $(b x-\alpha(x) b) V=\{0\}$ for all $x \in R$. Since $V$ is faithful it follows that $b x-\alpha(x) b=0$ for all $x \in R$ and we are done.

Thus, there exists $v_{0} \in V$ such that $v_{0}$ and $T^{-1} c v_{0}$ are linearly $D$-independent. If $\operatorname{dim}_{D} V \geq 3$, then there exists $w \in V$ such that $w, v$, and $T^{-1} b v$ are linearly $D$-independent. We will denote $T^{-1} b v=u$. By the density of $Q$ there exist $r_{1}, r_{2}, r_{3} \in Q$ such that

$$
r_{1} v=v, r_{2} v=v, r_{1} u=0, r_{2} u=w, r_{1} w=u .
$$


Thus, by (16) we have the contradiction

$$
0=\Phi\left(r_{1}, r_{2}\right) v=(-\gamma)^{r\left(p^{t}-1\right)} b v \neq 0 .
$$

Hence, we may consider the last case that $\operatorname{dim}_{D} V=2$. Then $Q$ is a finitedimensional central simple algebra over $C$ since $D$ is finite-dimensional over $C$. Moreover, if $C$ is finite, then $D$ is finite. Thus, $D$ is a commutative field and we are done. So, we may assume that $C$ is infinite. We will denote

$$
\begin{aligned}
& \Phi_{0}=-\left[x_{1}, x_{2}\right]^{m} b\left[x_{1}, x_{2}\right]^{n+r}-\left[x_{1}, x_{2}\right]^{m+n} b\left[x_{1}, x_{2}\right]^{r}, \\
& \Phi_{1}=\alpha\left(\left[x_{1}, x_{2}\right]^{m}\right) b\left[x_{1}, x_{2}\right]^{n+r}, \\
& \Phi_{2}=\left[x_{1}, x_{2}\right]^{m} \alpha\left(\left[x_{1}, x_{2}\right]^{n}\right) b\left[x_{1}, x_{2}\right]^{r}, \\
& \Phi_{3}=\left[x_{1}, x_{2}\right]^{m+n} \alpha\left(\left[x_{1}, x_{2}\right]^{r}\right) b, \\
& \Phi_{4}=-\alpha\left(\left[x_{1}, x_{2}\right]^{m+n+r}\right) b,
\end{aligned}
$$

and from (15) we have that $Q$ satisfies

$$
\Phi_{0}+\lambda_{1} \Phi_{1}+\lambda_{2} \Phi_{2}+\lambda_{3} \Phi_{3}+\lambda_{4} \Phi_{4}=0
$$

for all $0 \neq \gamma \in C$. Here, $\lambda_{1}=\gamma^{m\left(p^{t}-1\right)}, \lambda_{2}=\gamma^{n\left(p^{t}-1\right)}, \lambda_{3}=\gamma^{r\left(p^{t}-1\right)}$, and $\lambda_{4}=\gamma^{(m+n+r)\left(p^{t}-1\right)}$. Replacing $\gamma$ successively by $1, \gamma^{2}, \gamma^{3}, \gamma^{4}$ the identity (17) gives the homogeneous system of equations:

$$
\left\{\begin{array}{l}
\Phi_{0}+\Phi_{1}+\Phi_{2}+\Phi_{3}+\Phi_{4}=0 \\
\Phi_{0}+\lambda_{1} \Phi_{1}+\lambda_{2} \Phi_{2}+\lambda_{3} \Phi_{3}+\lambda_{4} \Phi_{4}=0 \\
\Phi_{0}+\lambda_{1}^{2} \Phi_{1}+\lambda_{2}^{2} \Phi_{2}+\lambda_{3}^{2} \Phi_{3}+\lambda_{4}^{2} \Phi_{4}=0 \\
\Phi_{0}+\lambda_{1}^{3} \Phi_{1}+\lambda_{2}^{3} \Phi_{2}+\lambda_{3}^{3} \Phi_{3}+\lambda_{4}^{3} \Phi_{4}=0 \\
\Phi_{0}+\lambda_{1}^{4} \Phi_{1}+\lambda_{2}^{4} \Phi_{2}+\lambda_{3}^{4} \Phi_{3}+\lambda_{4}^{4} \Phi_{4}=0
\end{array}\right.
$$

Moreover, since $C$ is infinite, there exists infinitely many $\gamma \in C$ such that $\gamma^{h\left(p^{t}-1\right)} \neq 1$ for $h=1, \ldots, m+n+r$. Hence, the Vandermonde determinant

$$
\left|\begin{array}{ccccc}
1 & 1 & 1 & 1 & 1 \\
1 & \lambda_{1} & \lambda_{2} & \lambda_{3} & \lambda_{4} \\
1 & \lambda_{1}^{2} & \lambda_{2}^{2} & \lambda_{3}^{2} & \lambda_{4}^{2} \\
1 & \lambda_{1}^{3} & \lambda_{2}^{3} & \lambda_{3}^{3} & \lambda_{4}^{3} \\
1 & \lambda_{1}^{4} & \lambda_{2}^{4} & \lambda_{3}^{4} & \lambda_{4}^{4}
\end{array}\right|= \pm\left(1-\lambda_{1}\right) \cdot \prod_{1 \leq i<j \leq 4}\left(\lambda_{i}-\lambda_{j}\right)
$$

is not zero. Thus, we can solve the above system of equations and obtain $\Phi=0$. Hence, $Q$ satisfies

$$
\left[x_{1}, x_{2}\right]^{m} b\left[x_{1}, x_{2}\right]^{n+r}+\left[x_{1}, x_{2}\right]^{m+n} b\left[x_{1}, x_{2}\right]^{r}=0
$$

and, by Lemma 2.1, $Q$ is commutative, a contradiction. The proof of proposition is completed. 


\section{The proof of Theorem 1.2}

We are now ready to prove the main result of this paper. So, let $m, n, r$ be nonzero fixed positive integers, $R$ a 2-torsion free prime ring, $L$ a non-central Lie ideal of $R, D: R \longrightarrow R$ a skew derivations of $R$, and

$$
E(x)=D\left(x^{m+n+r}\right)-D\left(x^{m}\right) x^{n+r}-x^{m} D\left(x^{n}\right) x^{r}-x^{m+n} D\left(x^{r}\right), x \in R .
$$

We have to prove that if $E(x)=0$ for all $x \in L$, then $D$ is a usual derivation of $R$ or $R$ satisfies $s_{4}\left(x_{1}, \ldots, x_{4}\right)$, the standard identity of degree 4 .

Let $\alpha \in \operatorname{Aut}(R)$ such that $D(x y)=D(x) y+\alpha(x) D(y)$ for all $x, y \in R$. In the case $\alpha=1$, the identity map of $R$, there is nothing to prove. Hence, we may assume that $\alpha \neq 1$.

We will divide the proof into two parts. Firstly, consider the case when $D$ is inner, i.e., there exists $b \in Q$ such that $D(x)=b x-\alpha(x) b$ for all $x \in R$. In the light of Proposition 3.1 we have that either $D=0$ or $R$ satisfies $s_{4}\left(x_{1}, \ldots, x_{4}\right)$ and we are done.

Now, assume that $D$ is outer. As above, there exists a suitable two-sided ideal $I$ of $R$ such that $0 \neq[I, R] \subseteq L$. Hence, by (4), $I$ satisfies

$$
\begin{gathered}
\left(\alpha\left(\left[x_{1}, x_{2}\right]^{m}\right)-\left[x_{1}, x_{2}\right]^{m}\right) D\left(\left[x_{1}, x_{2}\right]^{n}\right)\left[x_{1}, x_{2}\right]^{r} \\
+\left(\alpha\left(\left[x_{1}, x_{2}\right]^{m+n}\right)-\left[x_{1}, x_{2}\right]^{m+n}\right) D\left(\left[x_{1}, x_{2}\right]^{r}\right)=0 .
\end{gathered}
$$

Since by [5, Theorem 2] $I, R$, and $Q$ satisfy the same generalized polynomial identities with a single skew derivation, $Q$ satisfies the identity(18) as well. Note that for $1<n \in \mathbb{N}$

$$
D\left(x^{n}\right)=\sum_{i=0}^{n-1} \alpha\left(x^{i}\right) D(x) x^{n-i-1}, x \in R
$$

and

$$
D\left(\left[x_{1}, x_{2}\right]\right)=D\left(x_{1}\right) x_{2}+\alpha\left(x_{1}\right) D\left(x_{2}\right)-D\left(x_{2}\right) x_{1}-\alpha\left(x_{2}\right) D\left(x_{1}\right), x_{1}, x_{2} \in R .
$$

By (18) we obtain

$$
\begin{aligned}
& \alpha\left(\left[x_{1}, x_{2}\right]^{m}\right)\left(\sum_{i=0}^{n-1} \alpha\left(\left[x_{1}, x_{2}\right]^{i}\right)\left(D\left(x_{1}\right) x_{2}+\alpha\left(x_{1}\right) D\left(x_{2}\right)-D\left(x_{2}\right) x_{1}-\alpha\left(x_{2}\right) D\left(x_{1}\right)\right)\left[x_{1}, x_{2}\right]^{n-i-1}\right)\left[x_{1}, x_{2}\right]^{r} \\
& -\left[x_{1}, x_{2}\right]^{m}\left(\sum_{i=0}^{n-1} \alpha\left(\left[x_{1}, x_{2}\right]^{i}\right)\left(D\left(x_{1}\right) x_{2}+\alpha\left(x_{1}\right) D\left(x_{2}\right)-D\left(x_{2}\right) x_{1}-\alpha\left(x_{2}\right) D\left(x_{1}\right)\right)\left[x_{1}, x_{2}\right]^{n-i-1}\right)\left[x_{1}, x_{2}\right]^{r} \\
& +\alpha\left(\left[x_{1}, x_{2}\right]^{m+n}\right)\left(\sum_{j=0}^{r-1} \alpha\left(\left[x_{1}, x_{2}\right]^{j}\right)\left(D\left(x_{1}\right) x_{2}+\alpha\left(x_{1}\right) D\left(x_{2}\right)-D\left(x_{2}\right) x_{1}-\alpha\left(x_{2}\right) D\left(x_{1}\right)\right)\left[x_{1}, x_{2}\right]^{r-j-1}\right) \\
& -\left[x_{1}, x_{2}\right]^{m+n}\left(\sum_{j=0}^{r-1} \alpha\left(\left[x_{1}, x_{2}\right]^{j}\right)\left(D\left(x_{1}\right) x_{2}+\alpha\left(x_{1}\right) D\left(x_{2}\right)-D\left(x_{2}\right) x_{1}-\alpha\left(x_{2}\right) D\left(x_{1}\right)\right)\left[x_{1}, x_{2}\right]^{r-j-1}\right)=0
\end{aligned}
$$

for all $x_{1}, x_{2} \in Q$. Since $D$ is outer and by [5], $Q$ satisfies

$$
\alpha\left(\left[x_{1}, x_{2}\right]^{m}\right)\left(\sum_{i=0}^{n-1} \alpha\left(\left[x_{1}, x_{2}\right]^{i}\right)\left(y_{1} x_{2}+\alpha\left(x_{1}\right) y_{2}-y_{2} x_{1}-\alpha\left(x_{2}\right) y_{1}\right)\left[x_{1}, x_{2}\right]^{n-i-1}\right)\left[x_{1}, x_{2}\right]^{r}
$$




$$
\begin{aligned}
& -\left[x_{1}, x_{2}\right]^{m}\left(\sum_{i=0}^{n-1} \alpha\left(\left[x_{1}, x_{2}\right]^{i}\right)\left(y_{1} x_{2}+\alpha\left(x_{1}\right) y_{2}-y_{2} x_{1}-\alpha\left(x_{2}\right) y_{1}\right)\left[x_{1}, x_{2}\right]^{n-i-1}\right)\left[x_{1}, x_{2}\right]^{r} \\
& +\alpha\left(\left[x_{1}, x_{2}\right]^{m+n}\right)\left(\sum_{j=0}^{r-1} \alpha\left(\left[x_{1}, x_{2}\right]^{j}\right)\left(y_{1} x_{2}+\alpha\left(x_{1}\right) y_{2}-y_{2} x_{1}-\alpha\left(x_{2}\right) y_{1}\right)\left[x_{1}, x_{2}\right]^{r-j-1}\right) \\
& -\left[x_{1}, x_{2}\right]^{m+n}\left(\sum_{j=0}^{r-1} \alpha\left(\left[x_{1}, x_{2}\right]^{j}\right)\left(y_{1} x_{2}+\alpha\left(x_{1}\right) y_{2}-y_{2} x_{1}-\alpha\left(x_{2}\right) y_{1}\right)\left[x_{1}, x_{2}\right]^{r-j-1}\right)=0 .
\end{aligned}
$$

Moreover, if $\alpha$ is outer, by [5] and identity (19), $Q$ satisfies

$$
\begin{gathered}
{\left[z_{1}, z_{2}\right]^{m}\left(\sum_{i=0}^{n-1}\left[z_{1}, z_{2}\right]^{i}\left(y_{1} x_{2}+z_{1} y_{2}-y_{2} x_{1}-z_{2} y_{1}\right)\left[x_{1}, x_{2}\right]^{n-i-1}\right)\left[x_{1}, x_{2}\right]^{r}} \\
-\left[x_{1}, x_{2}\right]^{m}\left(\sum_{i=0}^{n-1}\left[z_{1}, z_{2}\right]^{i}\left(y_{1} x_{2}+z_{1} y_{2}-y_{2} x_{1}-z_{2} y_{1}\right)\left[x_{1}, x_{2}\right]^{n-i-1}\right)\left[x_{1}, x_{2}\right]^{r} \\
+\left[z_{1}, z_{2}\right]^{m+n}\left(\sum_{j=0}^{r-1}\left[z_{1}, z_{2}\right]^{j}\left(y_{1} x_{2}+z_{1} y_{2}-y_{2} x_{1}-z_{2} y_{1}\right)\left[x_{1}, x_{2}\right]^{r-j-1}\right) \\
-\left[x_{1}, x_{2}\right]^{m+n}\left(\sum_{j=0}^{r-1}\left[z_{1}, z_{2}\right]^{j}\left(y_{1} x_{2}+z_{1} y_{2}-y_{2} x_{1}-z_{2} y_{1}\right)\left[x_{1}, x_{2}\right]^{r-j-1}\right)=0 .
\end{gathered}
$$

In particular, if we write $z_{1}=z_{2}=0, y_{1}=x_{1}$, and $y_{2}=x_{2}$ in $(20)$, we get that $Q$ satisfies

$$
-2\left[x_{1}, x_{2}\right]^{m+n+r}=0 .
$$

In other words, $Q$ is commutative (see [7] for a fixed bounded index of nilpotency) and we are done.

At the end we have to consider the case when $\alpha$ is inner. So, there exists an invertible element $q \in Q$ such that $\alpha(x)=q x q^{-1}$ for all $x \in R$. Writing $y_{1}=0$ and $y_{2}=q y_{3}$ in (19) we obtain

$$
\begin{aligned}
& \left(q\left[x_{1}, x_{2}\right]^{m} q^{-1}-\left[x_{1}, x_{2}\right]^{m}\right)\left(\sum_{i=0}^{n-1}\left(q\left[x_{1}, x_{2}\right]^{i} q^{-1}\right)\left(q\left[x_{1}, y_{3}\right]\right)\left[x_{1}, x_{2}\right]^{n-i-1}\right)\left[x_{1}, x_{2}\right]^{r} \\
& \left(q\left[x_{1}, x_{2}\right]^{m+n} q^{-1}-\left[x_{1}, x_{2}\right]^{m+n}\right)\left(\sum_{j=0}^{r-1}\left(q\left[x_{1}, x_{2}\right]^{j} q^{-1}\right)\left(q\left[x_{1}, y_{3}\right]\right)\left[x_{1}, x_{2}\right]^{r-j-1}\right)=0
\end{aligned}
$$

for all $x_{1}, x_{2}, y_{3} \in Q$. Denote the left hand side of the identity (21) by $P\left(x_{1}, x_{2}, y_{3}\right)$. Note that $q \notin C$ since $\alpha \neq 1$. Therefore, (21) is a non-trivial generalized polynomial identity for $Q$. By Martindale's theorem [12], $Q$ is a primitive ring having a nonzero socle with $C$ as the associated division ring. In the light of Jacobson's theorem $[9$, p. 75$]$ a ring $R$ is isomorphic to a dense ring of linear transformations on some vector space $V$ over $C$. Of course, we may assume that $\operatorname{dim}_{C} V \geq 3$. 
Since $q \notin C$ there exists $v \in V$ such that $v$ and $q v$ are linearly $C$-independent. Moreover, since $\operatorname{dim}_{C} V \geq 3$ we can find $w \in V$ such that $\{v, q v, w\}$ are linearly $C$-independent.

Assume first that $r \geq 2$. By the density of $Q$, there exist $r_{1}, r_{2}, r_{3} \in Q$ such that

$$
\begin{gathered}
r_{1} v=0, \quad r_{2} v=w, \\
r_{1} q v=w, \quad r_{2} q v=0, \quad r_{3} q v=0, \\
r_{1} w=v, \quad r_{2} w=0, \quad r_{3} w=-v .
\end{gathered}
$$

Then $\left[r_{1}, r_{2}\right] v=0,\left[r_{1}, r_{2}\right] q v=0,\left[r_{1}, r_{3}\right] q v=v$. This yields that

$$
0=P\left(r_{1}, r_{2}, r_{3}\right) q v=q v \neq 0,
$$

a contradiction. On the other hand, if $r=1$, we can write (21) as follows

$$
\begin{gathered}
\left(q\left[x_{1}, x_{2}\right]^{m} q^{-1}-\left[x_{1}, x_{2}\right]^{m}\right)\left(\sum_{i=0}^{n-1}\left(q\left[x_{1}, x_{2}\right]^{i} q^{-1}\right)\left(q\left[x_{1}, y_{3}\right]\right)\left[x_{1}, x_{2}\right]^{n-i-1}\right)\left[x_{1}, x_{2}\right] \\
+\left(q\left[x_{1}, x_{2}\right]^{m+n} q^{-1}-\left[x_{1}, x_{2}\right]^{m+n}\right) q\left[x_{1}, y_{3}\right]=0 .
\end{gathered}
$$

By the density of $Q$, there exist $r_{1}, r_{2}, r_{3} \in Q$ such that

$$
\begin{gathered}
r_{1} v=0, \quad r_{2} v=0, \quad r_{3} v=w, \\
r_{1} q v=w, \quad r_{2} q v=0, \quad r_{3} q v=0, \\
r_{1} w=v, \quad r_{2} w=-q v .
\end{gathered}
$$

Then $\left[r_{1}, r_{2}\right] v=0,\left[r_{1}, r_{2}\right] q v=q v,\left[r_{1}, r_{3}\right] v=v$. This yields

$$
0=P\left(r_{1}, r_{2}, r_{3}\right) v=-q v \neq 0,
$$

a contradiction. The proof of Theorem 1.2 is completed.

At the end we will give an example which shows that in our main theorem we can not expect the conclusion that $R$ is a commutative ring.

Example 4.1. Let $R$ be a ring of all $2 \times 2$ matrices over the field of complex numbers and let $\alpha: R \rightarrow R$ be an automorphism of $R$ defined by $\alpha(x)=q x q^{-1}$ for all $x \in R$ and some fixed invertible element $q \in R$. Let $b \in R$ be a fixed nonzero matrix. Consider a skew derivation $D: R \rightarrow R$ defined by $D(x)=b x-\alpha(x) b$ for all $x \in R$. Let $L=[R, R]$. Then $u^{2} \in Z(R)$ for all $u \in L$. Hence, for $m=r=2$ we have $\alpha\left(u^{m}\right)-u^{m}=q u^{2} q^{-1}-u^{2}=0$ and $b u^{r}-\alpha\left(u^{r}\right) b=b u^{2}-q u^{2} q^{-1} b=b u^{2}-u^{2} b=0$. Therefore, the hypothesis of Theorem 1.2 are satisfied. Note also that $R$ satisfies $s_{4}\left(x_{1}, \ldots, x_{4}\right)$ but it is not a commutative ring. 


\section{References}

[1] K. I. Beidar, W. S. Martindale III, and A. V. Mikhalev, Rings with Generalized Identities, Pure and Applied Math., Dekker, New York, 1996.

[2] C.-L. Chuang, GPIs having coefficients in Utumi quotient rings, Proc. Amer. Math. Soc. 103 (1988), no. 3, 723-728.

[3] _ Differential identities with automorphisms and antiautomorphisms I, J. Algebra 149 (1992), no. 2, 371-404.

[4] _ Differential identities with automorphisms and antiautomorphisms II, J. Algebra 160 (1993), no. 1, 130-171.

[5] C.-L. Chuang and T.-K. Lee, Identities with a single skew derivation, J. Algebra 288 (2005), no. 1, 59-77.

[6] B. Dhara, V. De Filippis, and R. K. Sharma, Generalized derivations and left multipliers on Lie ideals, Aequationes Math. 81 (2011), 251-261.

[7] O. M. Di Vincenzo, A note on $k$-th commutators in an associative ring, Rend. Circ. Mat. Palermo (2) 47 (1998), no. 1, 106-112.

[8] V. K. Harčenko, Generalized identities with automorphisms, Algebra i Logika 14 (1975), no. 2, 215-237.

[9] N. Jacobson, Structure of Rings, Amer. Math. Soc., Providence, 1964.

[10] C. Lanski, Differential identities, Lie ideals, and Posner's theorems, Pacific J. Math. 134 (1988), no. 2, 275-297.

[11] T.-K. Lee and K.-S. Liu, Generalized skew derivations with algebraic values of bounded degree, preprint.

[12] W. S. Martindale III, Prime rings satisfying a generalized polynomial identity, J. Algebra 12 (1969), 576-584.

VinCENZO De FiLipPis

DI.S.I.A.

Faculty of Engineering University of Messina

Contrada Di Dio, 98166, Messina, Italy

E-mail address: defilippis@unime.it

AJDA FOŠNER

Faculty of Management

UNIVERSITY OF PRIMORSKA

Cankarjeva 5, SI-6104 Koper, Slovenia

E-mail address: ajda.fosner@fm-kp.si 